\title{
Single-Pulse Transcranial Magnetic Stimulation-Evoked Potential Amplitudes and Latencies in the Motor and Dorsolateral Prefrontal Cortex among Young, Older Healthy Participants, and Schizophrenia Patients
}

\author{
Yoshihiro Noda ${ }^{1} *{ }^{(D)}$, Mera S. Barr ${ }^{2}$, Reza Zomorrodi ${ }^{3}$, Robin F. H. Cash ${ }^{4}$, Pantelis Lioumis ${ }^{5}$, Robert Chen ${ }^{6}$ (D) \\ Zafiris J. Daskalakis ${ }^{7}$ and Daniel M. Blumberger ${ }^{2,3,8}$
}

1 Department of Neuropsychiatry, Graduate School of Medicine, Keio University School of Medicine, Tokyo 160-8582, Japan

2 Department of Psychiatry, University of Toronto, Toronto, ON M5T 1R8, Canada; mera.barr@gmail.com (M.S.B.); Daniel.Blumberger@camh.ca (D.M.B.)

3 Temerty Centre for Therapeutic Brain Intervention, Centre for Addiction and Mental Health, Toronto, ON M6J 1H4, Canada; Reza.Zomorrodi@camh.ca

4 Monash Alfred Psychiatry Research Centre, Monash University Central Clinical School and the Alfred, Melbourne 3004, Australia; robin.cash2@gmail.com

5 Department of Neuroscience and Biomedical Engineering, Aalto University School of Science, FI-00076 Espoo, Finland; plioumis@gmail.com

check for

updates

Citation: Noda, Y.; Barr, M.S.;

Zomorrodi, R.; Cash, R.F.H.; Lioumis,

P.; Chen, R.; Daskalakis, Z.J.;

Blumberger, D.M. Single-Pulse

Transcranial Magnetic

Stimulation-Evoked Potential

Amplitudes and Latencies in the

Motor and Dorsolateral Prefrontal

Cortex among Young, Older Healthy

Participants, and Schizophrenia

Patients. J. Pers. Med. 2021, 11, 54.

https://doi.org/10.3390/

jpm11010054

Received: 19 December 2020

Accepted: 15 January 2021

Published: 17 January 2021

Publisher's Note: MDPI stays neutral with regard to jurisdictional clai$\mathrm{ms}$ in published maps and institutional affiliations.

Copyright: (C) 2021 by the authors. Licensee MDPI, Basel, Switzerland. This article is an open access article distributed under the terms and conditions of the Creative Commons Attribution (CC BY) license (https:// creativecommons.org/licenses/by/ $4.0 /)$.
6 Division of Neurology, Department of Medicine, University of Toronto, Division of Brain, Imaging and Behaviour-Systems Neuroscience, Krembil Research Institute, University Health Network, Toronto, ON M5T 2S8, Canada; robert.chen@uhn.ca

7 Department of Psychiatry, UC San Diego Health, San Diego, CA 92093, USA; zdaskalakis@health.ucsd.edu

8 Campbell Family Mental Health Research Institute, Centre for Addiction and Mental Health, Toronto, ON M5T 1R8, Canada

* Correspondence: yoshi-tms@keio.jp; Tel.: +81-3-3353-1211 (ext. 61857)

\begin{abstract}
Background: The combination of transcranial magnetic stimulation (TMS) with electroencephalography (EEG) allows for non-invasive investigation of cortical response and connectivity in human cortex. This study aimed to examine the amplitudes and latencies of each TMS-evoked potential (TEP) component induced by single-pulse TMS (spTMS) to the left motor (M1) and dorsolateral prefrontal cortex (DLPFC) among healthy young participants (YNG), older participants (OLD), and patients with schizophrenia (SCZ). Methods: We compared the spatiotemporal characteristics of TEPs induced by spTMS among the groups. Results: Compared to YNG, M1-spTMS induced lower amplitudes of N45 and P180 in OLD and a lower amplitude of P180 in SCZ, whereas the DLPFC-spTMS induced a lower N45 in OLD. Further, OLD demonstrated latency delays in P60 after M1-spTMS and in N45-P60 over the right central region after left DLPFC-spTMS, whereas SCZ demonstrated latency delays in N45-P60 over the midline and right central regions after DLPFC-spTMS. Conclusions: These findings suggest that inhibitory and excitatory mechanisms mediating TEPs may be altered in OLD and SCZ. The amplitude and latency changes of TEPs with spTMS may reflect underlying neurophysiological changes in OLD and SCZ, respectively. The spTMS administered to M1 and the DLPFC can probe cortical functions by examining TEPs. Thus, TMS-EEG can be used to study changes in cortical connectivity and signal propagation from healthy to pathological brains.
\end{abstract}

Keywords: TMS-EEG; TMS-evoked potentials; dorsolateral prefrontal cortex; motor cortex; schizophrenia

\section{Highlights}

- $\quad$ The single-pulse transcranial magnetic stimulation (TMS) (spTMS) allows probing and visualization of the spatiotemporal characteristics of each young (YNG), older (OLD), and schizophrenia (SCZ) group. 
- Different TMS-evoked potential (TEP) characteristics induced by spTMS were observed in the OLD and SCZ compared with YNG.

- $\quad$ Specifically, changes in P30 and N45 deflections of TEPs by spTMS were important in classifying these groups across the motor cortex (M1) and dorsolateral prefrontal cortex (DLPFC).

\section{Introduction}

Electroencephalography (EEG) studies probed by transcranial magnetic stimulation (TMS) have become feasible since Ilmoniemi et al. initially developed the technology of the TMS-compatible EEG system [1]. One advantage of the TMS-EEG paradigm is that it allows for the investigation and mapping of cortical regions without the need to administer perception stimuli (e.g., visual, auditory, sensory stimuli) or specific cognitive tasks as in event-related potential studies. The TMS-EEG technique has been applied in several research labs worldwide mainly for healthy participants [2-8], and previous studies using TMS-EEG have demonstrated the typical pattern of TMS-evoked potentials (TEPs) and their topographical distribution [9-12]. Specifically, single-pulse TMS (spTMS) to the primary motor cortex (M1) as well as the dorsolateral prefrontal cortex (DLPFC) induces a wellcharacterized series of TEP components of P30, N45, P60, N100, and P180 [9,10,13], that are highly reproducible [10] and the amplitudes of these TEP components vary depending on the intensity of TMS $[14,15]$. Individual TEP components are thought to be mediated by distinct inhibitory and excitatory processes. Moreover, it has been reported that amplitudes of TEPs elicited by spTMS vary depending on the stimulated area [16], notable being reduced at the DLPFC compared to M1-spTMS [17,18].

A previous spTMS-EEG study investigated the topographical distribution pattern and amplitude power of TEPs after left superior frontal cortex stimulation between healthy young (YNG) and older (OLD) participants compared to patients with Alzheimer's disease [19]. The study demonstrated no significant difference in the topographical distribution of TEPs or amplitude power of early TEP components (N45 to N100) between healthy YNG and OLD participants. However, significantly smaller distribution area and power of the early TEPs were observed among patients with Alzheimer's disease compared to healthy young participants. The other spTMS-EEG studies examined spectrum power and time-frequency analyses of TEPs after premotor cortex stimulation between patients with schizophrenia (SCZ) and healthy participants, which demonstrated a significantly smaller early TEP power [20] and reduced amplitude of TMS-induced gamma oscillations and its effective connectivity [21] in patients with SCZ compared to the healthy participants.

In OLD adults as well as in patients with SCZ, it is known that atrophy of the cerebral cortex is associated with aging [22] or disease progression [23]. Further, age and neuropathological changes may contribute to decreased nerve conduction velocity [24-26]. Therefore, it is speculated that TEP responses by spTMS, especially in latency, may be reduced in these populations compared to younger healthy adults. Thus, it may be possible to probe these physiological and pathological alterations using spTMS-EEG [27].

To our knowledge, there are a limited number of studies [19-21,28] examining the spatiotemporal patterns of TEP components by spTMS in OLD adults and patients with SCZ compared to YNG adults, specifically investigating the TEPs with stimulation of the DLPFC, which is an area more directly associated with the changes that occur during healthy aging as well as the pathophysiology of SCZ [29,30]. It is therefore important to identify the neurophysiological profile of TEPs by spTMS among healthy and pathological brains to better understand the pathophysiology of neuropsychiatric disorders [31,32]. Based on the previous findings that showed reduced TEP amplitude with age [19-21,28], we hypothesized that the OLD group and SCZ group would have significantly lower amplitudes of TEPs and more delayed latencies of TEPs compared to those of the YNG group. Thus, we aimed to investigate the amplitudes and latencies of TEPs by spTMS to M1 as well as the DLPFC in an exploratory fashion from both the stimulated region (i.e., M1 
and DLPFC) and other connected regions of interest (ROI: contralateral M1, DLPFC, and midline central area) among the groups (YNG, OLD, and SCZ).

\section{Materials and Methods}

\subsection{Participants}

Twelve right-handed YNG ( 6 female, mean age: $39 \pm 12$ yrs), 12 right-handed OLD (6 female, mean age: $72 \pm 9 \mathrm{yrs}$ ), and 12 right-handed patients with SCZ (4 female, mean age: $41 \pm 10 \mathrm{yrs}$ ) participated in this study. Participants of all groups were eligible to participate in this study if they met the following criteria: (i) between ages 18 and 59 for the YNG and SCZ, or ages above 60 for the OLD; (ii) no history of neurological disorders including seizure or stroke; (iii) no history of alcohol or other drug abuse/dependence; and (iv) not being a smoker.

In addition, the YNG and OLD also satisfied with the following criteria: (v) no history of neuropsychiatric disorders; (vi) normal cognitive function assessed by neurocognitive battery; (vii) no prescription medications; while patients with SCZ met the following criteria; (viii) not taking benzodiazepines and anticholinergics. All participants were screened with the Structured Clinical Interview for DSM-IV Axis I Disorders or the MiniInternational Neuropsychiatric Interview prior to study participation. The present study was performed according to the Declaration of Helsinki and was reviewed and approved by the Research Ethics Board of the Centre for Addiction and Mental Health.

\subsection{TMS Procedure}

Monophasic TMS pulses were administered to the left M1 using a $70 \mathrm{~mm}$ figureof-eight coil, and a Magstim 200 stimulator (Magstim Company Ltd., Whitland, UK). During the TMS testing, the participants sat in a chair with their eyes open, and their bodies relaxed throughout the study. First, the M1 hotspot site for the right first dorsal interosseous muscle to evoke the largest motor evoked potential (MEP) with the lowest intensity was determined. Second, the individual intensity to induce $1 \mathrm{mV}$ peak-to-peak MEP amplitude of the same muscle was determined. The intensity to induce $1 \mathrm{mV}$ peakto-peak MEP amplitude was used for the spTMS in this study. At each stimulation site of M1 and DLPFC, 100 TMS pulses were applied per session, respectively. In addition, the stimulation site of the left DLPFC was identified using the EEG cap method. Specifically, the F5 electrode site was used as the target site of the left DLPFC according to our other studies (Noda et al., 2017a, Noda et al., 2017b).

\subsection{EEG Recording and Pre-Processing}

EEG was recorded through a 64-channel Neuroscan Synamps 2 with a TMS-compatible EEG cap (Compumedics Neuroscan, Victoria, Australia). Recording electrodes impedance was kept below $5 \mathrm{k} \Omega$ during the experiment. All electrodes were referenced to an electrode placed on the vertex. EEG signals were recorded at DC with a sampling rate of $20 \mathrm{kHz}$ and then an online lowpass filter of $200 \mathrm{~Hz}$ was applied. EEG data were processed offline using the MATLAB software (R2014a, The MathWorks, Natick, MA, USA).

\subsection{EEG Signal Processing}

EEG signal processing was performed in accordance to already published methodology (Noda et al., 2016). All EEG data were epoched from -1000 ms to $2000 \mathrm{~ms}$ relative to the TMS pulse. Baseline correction was performed with respect to the pre-stimulus interval $-500 \mathrm{~ms}$ to $-110 \mathrm{~ms}$. The epoched EEG data was re-segmented from $10 \mathrm{~ms}$ to $2000 \mathrm{~ms}$ post-TMS to limit the TMS-induced artifact. Then, EEG data were visually inspected to exclude trials and channels that were highly contaminated with noise. Subsequently, independent component analysis (ICA) was applied to minimize and remove the typical TMS-related decay artifacts as well as eye-related and muscle activity related components. Next, the Butterworth, zero-phase shift 1-55 Hz bandpass filter ( $24 \mathrm{~dB} / \mathrm{Oct}$ ) and notch filter were applied. Then, the processed data were downsampled to $1000 \mathrm{~Hz}$. In each subject, the 
number of ICA components that were removed from the original 62 ICA components was no greater than $20 \%$. Finally, data were re-referenced to the average reference for further analyses.

\subsection{Single-Pulses TMS-Evoked Potential (TEP) Analyses}

The TEP was analyzed individually focusing on the amplitude and latency of each TEP component (P30, N45, P60, N100, and P180) at the 5 ROIs (left frontal: AF3, F3, F5; right frontal: AF4, F4, F6; left central: C3, C5, CP5; right central: C4, C6, CP6; midline central: Fz, FCz, Cz; see Figure 1), which were obtained from M1-spTMS and DLPFC-spTMS experiments.
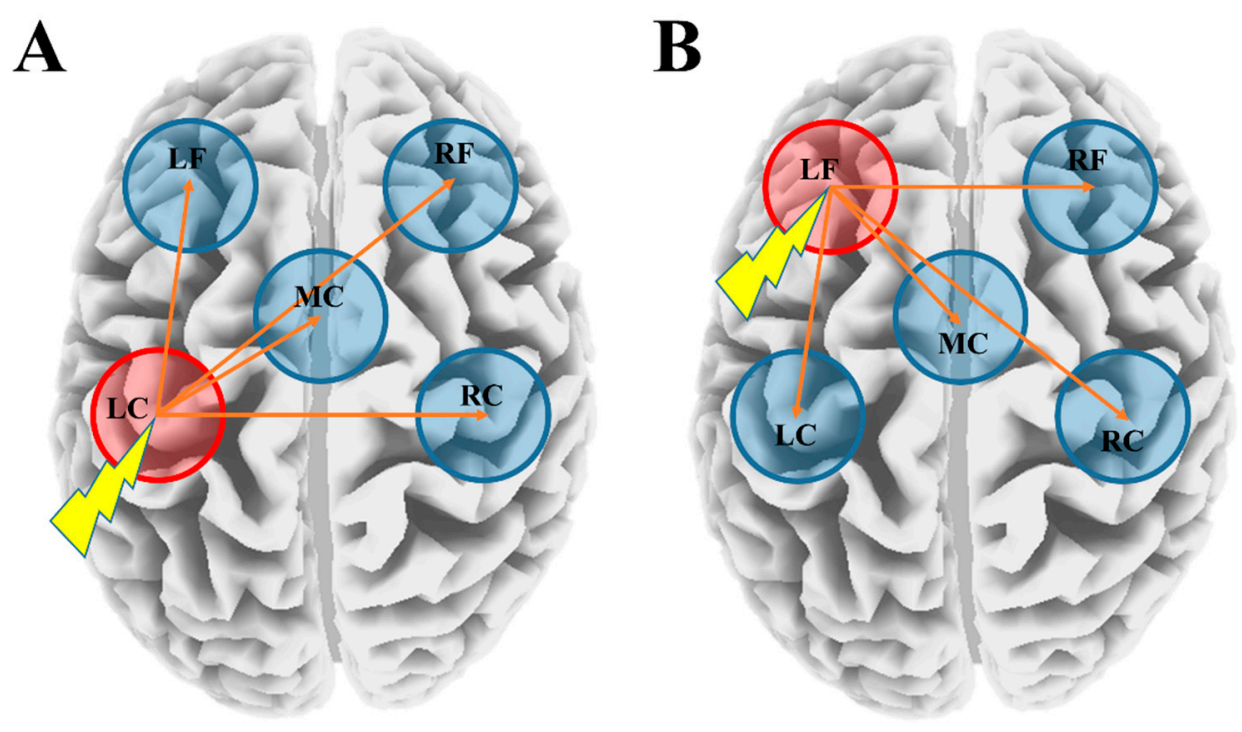

Figure 1. Schematic diagram of the transcranial magnetic stimulation (TMS) stimulation site and analysis site. (A) depicts single-pulse TMS (spTMS) administered to the left motor cortex (M1), while (B) shows spTMS applied to the left dorsolateral prefrontal cortex (DLPFC).

\subsection{Statistical Analyses}

The SPSS Statistics 19 (IBM, Armonk, New York, USA) was used for statistical analysis. In this study, normal distributions of the TEP data were confirmed with the Shapiro-Wilk test before performing the parametric statistical testing. For the analyses of TEP amplitudes, the analysis of variance (ANOVA) with TEP components (i.e., P30, N45, P60, N100, and P180) as a within-subject factor and groups (i.e., YNG, OLD, or SCZ) as a between-subject factor was applied for each M1-spTMS and the DLPFC-spTMS paradigm, separately. Here, we analyzed TEP data of M1-spTMS focusing on the left central (LC) ROI (i.e., left M1) while we analyzed TEP data of the DLPFC-spTMS focusing on the left frontal (LF) ROI (i.e., left DLPFC). When there was a significant interaction between the TEP component and group, post-hoc independent $t$-tests were applied to examine group differences of TEP amplitudes. Next, we conducted the multiple regression analysis with the group (i.e., YNG, OLD, or SCZ) as a dependent variable and the site of stimulation (i.e., M1 or DLPFC) and TEP component (i.e., P30, N45, P60, N100, and P180) as independent variables to explore the predictive factors to identify the groups using an enter method. In this regression analysis, we set a significant level of $\alpha=0.05 / 10$ (site of stimulations ${ }^{*} \mathrm{TEP}$ components) with Bonferroni correction.

For the analyses of TEP latencies, one-way ANOVAs and post-hoc paired t-tests were applied to explore the group difference of each TEP component for the same ROIs. Further, within-group level, the differences of TEP latencies between the LC ROI (i.e., the stimulated region of M1-spTMS) and other ROIs for M1-spTMS as well as between the 
LF ROI (i.e., the stimulated region of the DLPFC-spTMS) and other ROIs for the DLPFCspTMS. A significant level of $\alpha=0.05 / 25$ (site of ROIs*TEP components) with Bonferroni correction was applied here.

\section{Results}

\subsection{TEP Amplitude Differences between the YNG, OLD, and SCZ Groups}

With M1-spTMS, at the LC ROI (i.e., left M1), the ANOVA indicated a significant TEP-by-group interaction $\left(\mathrm{F}_{8,132}=4.504, p<0.0001\right)$. Subsequently, compared to the YNG group, post-hoc independent $\mathrm{t}$-tests revealed that the OLD group had significantly lower amplitudes of $\mathrm{N} 45\left(\mathrm{t}_{22}=-2.872, p=0.009\right.$; amplitude $\mathrm{N} 45$ at the LC in YNG $>$ amplitude $\mathrm{N} 45$ at the LC in OLD) and P180 ( $\mathrm{t}_{22}=5.283, p<0.0001$; amplitude P180 at the LC in YNG > amplitude P180 at the LC in OLD) TEPs, whereas the SCZ group had a significantly lower amplitude of P180 $\left(\mathrm{t}_{22}=4.394, p=0.0002\right.$; amplitude P180 at the LC in HC > amplitude P180 at the LC in SCZ). The results of TEPs after M1-spTMS are depicted in Figure 2A.
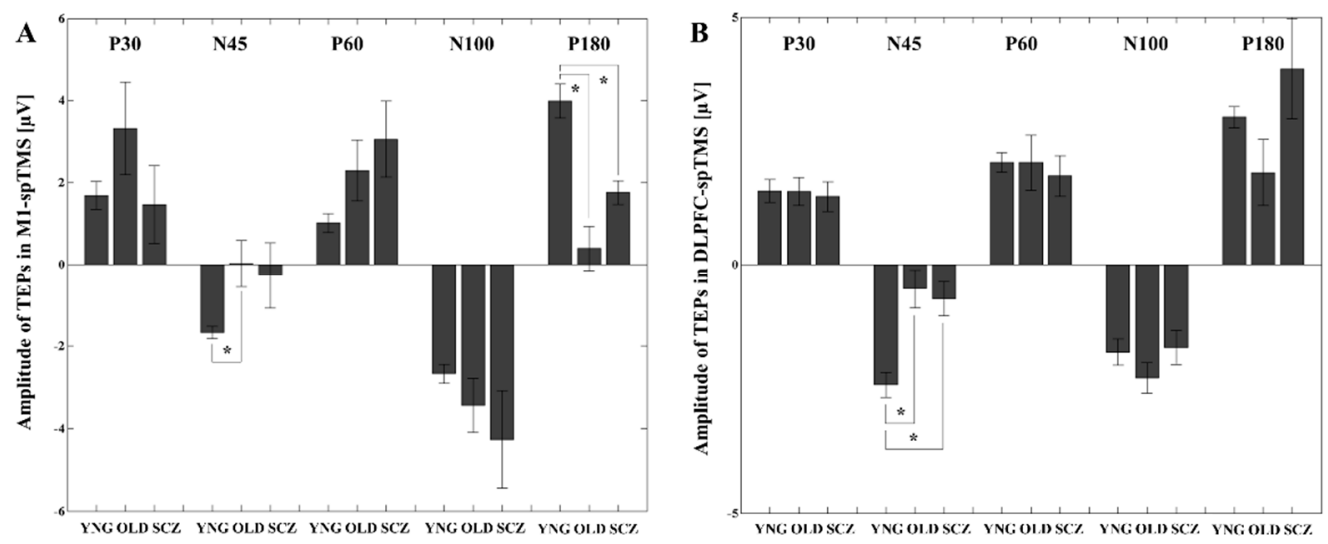

Figure 2. Amplitude differences for each TMS-evoked potential (TEP) component among healthy young (YNG), older (OLD), and patients with schizophrenia (SCZ) at the left central (LC) ROI (left M1). TEP amplitude differences among the groups at the left M1 induced by M1-spTMS were demonstrated in (A), while TEP amplitude differences among the groups over the left DLPFC induced by the DLPFC-spTMS are shown in (B). Compared to the YNG group, the OLD group had a significantly lower amplitude of the N45 TEP, while the OLD group and SCZ group had significantly lower amplitudes on the P180 TEP over the LC ROI (left M1) in M1-spTMS paradigm. In the DLPFC-spTMS, compared to the YNG group, the OLD group and SCZ group had significantly lower amplitudes on N45 TEP over the left frontal ROI (left DLPFC). * Significant differences.

On the other hand, at the LF ROI (i.e., left DLPFC) by the DLPFC-spTMS, the ANOVA showed a significant TEP-by-group interaction $\left(\mathrm{F}_{8,132}=2.599, p=0.011\right)$. Post-hoc independent $\mathrm{t}$-tests indicated that the OLD group had a significantly lower amplitude of N45 $\left(\mathrm{t}_{22}=-4.200, p=0.0004\right.$; amplitude N45 at the LF in YNG $>$ amplitude N45 at the LF in OLD), while the SCZ group also had a significantly lower amplitude of $\mathrm{N} 45\left(\mathrm{t}_{22}=-4.047\right.$, $p=0.001$; amplitude N45 at the LF in HC > amplitude N45 at the LF in SCZ), compared to the YNG group. The results of TEPs by the DLPFC-spTMS are described in Figure 2B.

Regarding P30 and N100 TEP amplitudes, no significant differences were observed among the three groups both in M1-spTMS or DLPFC-spTMS paradigm

Representative TEP waveforms from the left DLPFC obtained by spTMS for the YNG, OLD, and SCZ groups in this study are shown in the Supplementary Figure S1.

\subsection{Predictive Factors to Classify the Groups Based on the Multiple Regression Analysis}

The multiple regression analysis revealed that $\mathrm{P} 30$ and $\mathrm{N} 45$ components were significant factors (P30: $\beta=-0.410, p=0.004 ; \mathrm{N} 45: \beta=6.22, p<0.001 ; \mathrm{F} 6,65=4.825$, adjusted $\mathrm{R}^{2}$ $=0.244)$ to identify the difference between these groups. 


\subsection{Latency Differences for Each TEP between the YNG, OLD, and SCZ Groups}

With M1-spTMS, when we compared each TEP latency over all 5 ROIs between the YNG and OLD groups, the OLD group had a significantly longer P60 latency $\left(\mathrm{t}_{22}=-4.275\right.$, $p=0.0003$; latency P60 at the LC in YNG < latency P60 at the LC in OLD) over the LC ROI compared to the YNG group (see Figure 3A).
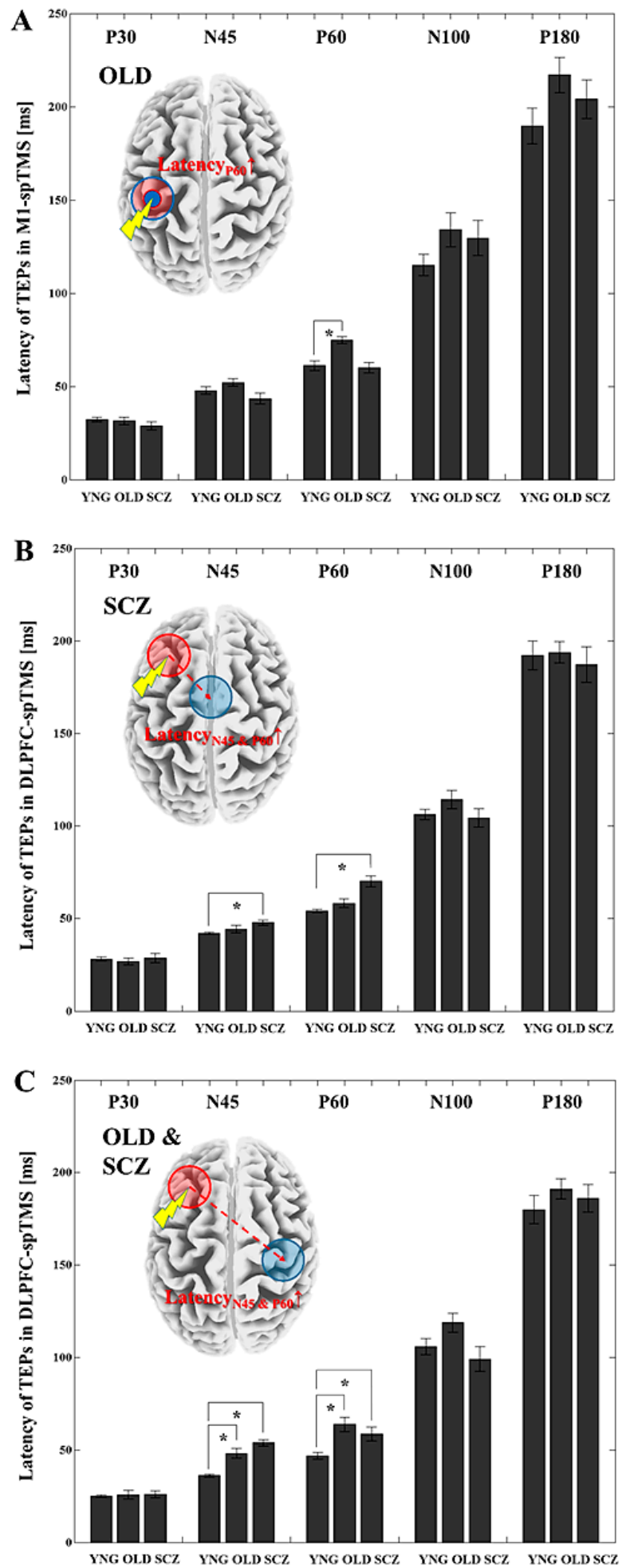

Figure 3. Latency differences for each TEP component among healthy young (YNG) participants, older (OLD) participants, and patients with schizophrenia (SCZ). TEP latency differences among groups at the LC ROI (left M1) induced by M1-spTMS 
were demonstrated in (A), while TEP latency differences among the groups induced by the DLPFC-spTMS are shown in (B) (over the midline central ROI) and (C) (over the right central ROI). In M1-spTMS, compared to the YNG group, the OLD group showed a significantly longer latency of the N45 TEP over the left M1 in M1-spTMS paradigm, whereas in the DLPFC-spTMS, the SCZ group showed significantly longer latencies of the N45 and P60 TEPs over the midline central ROI. Furthermore, after DLPFC-spTMS, the OLD group and SCZ group demonstrated significantly longer latencies of N45 and P60 TEPs over the right M1. In (A-C), a schematic diagram of a group showing significant findings is shown in the box.

* Significant differences.

Likewise, when we looked at the DLPFC-spTMS paradigm, the OLD group showed significantly longer N45 $\left(\mathrm{t}_{22}=-4.255, p=0.0003\right)$ and P60 $\left(\mathrm{t}_{22}=-4.033, p=0.0006\right)$ TEP latencies (latencies N45$\mathrm{P} 60$ at the RC in YNG < latencies N45-P60 at the RC in OLD) from the right central ROI compared to the YNG group. The SCZ group indicated significantly longer N45 $\left(t_{22}=-3.622, p=0.0015\right)$ and P60 $\left(\mathrm{t}_{22}=-5.095, p<0.0001\right)$ TEP latencies (latencies N45-P60 at the MC in $\mathrm{HC}<$ latencies N45-P60 at the $\mathrm{MC}$ in SCZ) at the midline central ROI (Figure 3B) as well as longer N45 (t22 $=-9.207, p<0.0001$ ) and P60 (P60: $\mathrm{t}_{22}=-2.945, p=0.007$ ) TEP latencies (latencies N45-P60 at the RC in HC < latency N45-P60 at the RC in SCZ) at the right central ROI (Figure 3C). For P30 and N100 TEP latencies, there were no significant differences among groups both in M1-spTMS or DLPFC-spTMS paradigm.

\subsection{Latency Differences between the Stimulated ROI and Other ROIs within the YNG, OLD, and SCZ Groups}

With M1-spTMS, there was no significant latency difference between the LC ROI (i.e., left M1) and other ROIs within groups. After DLPFC-spTMS, there was no significant difference in latencies between the LF ROI (i.e., left DLPFC) and other ROIs within the YNG or OLD group. However, within the SCZ group, there was a significant latency difference on $\mathrm{N} 45\left(\mathrm{t}_{11}=-4.326, p=0.0012\right.$; latency N45 at the LF in SCZ < latency N45 at the RC in SCZ) between the LF (i.e., left DLPFC) and right central ROIs (i.e., right M1), showing that the latency of N45 was significantly longer from the right central ROI compared to that of the left DLPFC (see Figure 4).

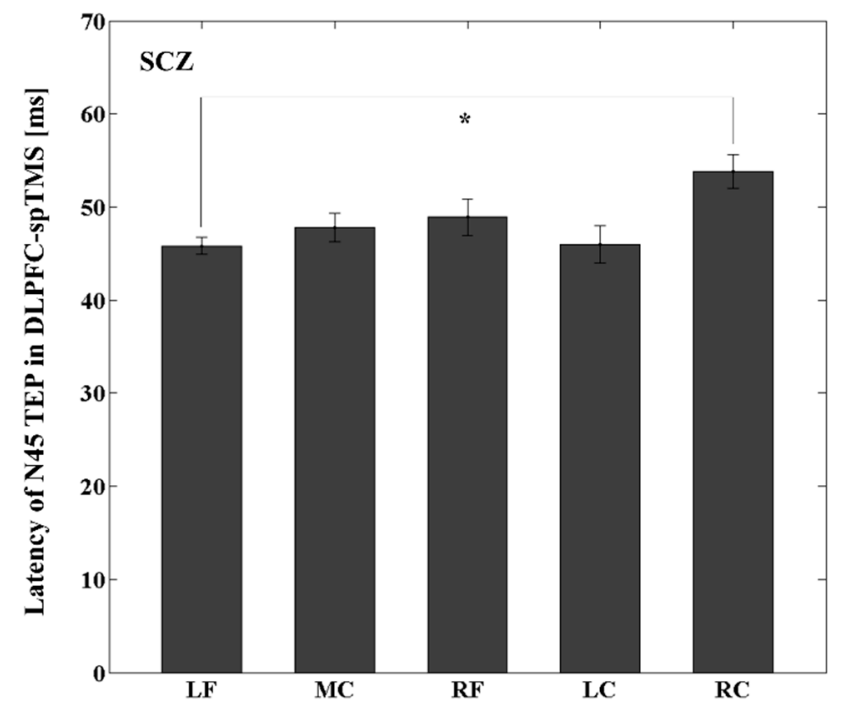

Figure 4. Latency differences between the left DLPFC and other ROIs within a schizophrenia (SCZ) group in the DLPFC-spTMS paradigm. There were no significant TEP latency differences between the left DLPFC and other ROIs within the young (YNG) and older (OLD) groups. However, within the SCZ group, the latency of N45 TEP was significantly longer over the right M1 compared to the left DLPFC. * Significant differences.

\section{Discussion}

The present study demonstrated unique spatiotemporal profiles of TEPs induced by spTMS of M1 and DLPFC. First, the OLD group demonstrated an attenuated response in the amplitude of N45 after M1-spTMS, which is in line with previous studies showing that there was a TEP amplitude reduction with age in healthy subjects [21,28], compared to the YNG group. Further, both the OLD and SCZ groups had attenuated responses in the amplitude of P180 after M1-spTMS, which is, at least in part, consistent with a previous study showing that SCZ had reduced spTMS-evoked gamma 
oscillations [21], as well as the amplitude of N45 by the DLPFC-spTMS, compared to those of the YNG group. Second, the OLD group showed a delayed response in the latency of P60 at the stimulated ROI after M1-spTMS compared to the YNG group. The SCZ group demonstrated a delayed response in the latency of N45 over the right central ROI within-subjects level as well as delayed responses in the latency of N45-P60 at the midline central ROI between-subjects level by the DLPFC-spTMS. In addition, the OLD and SCZ groups demonstrated a delayed response in the latency of N45-P60 over the right central ROI after DLPFC-spTMS compared to the YNG group.

To date, the origin and mechanisms of N45 and P180 have not been fully elucidated. However, previous studies demonstrated that $\mathrm{N} 45$ may be associated with gamma-aminobutyric acid receptor $A\left(\mathrm{GABA}_{\mathrm{A}}\right)$ mediated inhibition [7,33-35]. In the healthy YNG group, the amplitude of N45 component was relatively increased consistently across M1 and DLPFC by spTMS in our study (Figure 2). In contrast, P180 has been proposed to relate either to a late excitatory potential or cortical disinhibition. The latter interpretation may be more consistent with the GABAergic deficits shown in both aging and SCZ $[29,30]$. Thus, it seems that N45 may represent GABA $_{A}$ ergic inhibitory function and $\mathrm{P} 180$ may reflect more $\mathrm{GABA}_{\mathrm{A}}$ ergic disinhibition rather than just an excitatory potential itself.

In addition, several lines of evidence have shown that $\mathrm{GABA}_{\mathrm{A}}$ receptor-mediated inhibition in M1 as indexed by motor-evoked potentials decreases with age [36-38]. Further, magnetic resonance spectroscopic (MRS) studies have demonstrated that OLD participants have a significantly lower level of GABA compared to the young participants $[39,40]$. Therefore, these MRS findings appear at least partially consistent with TMS neurophysiological studies in OLD participants [29].

On the other hand, in patients with SCZ, GABAergic deficit is supported by neuropathological findings that demonstrate substantial reductions in GABAergic interneurons, GABA-synthesizing enzyme glutamic acid decarboxylase, and GABA-related gene expression in the cortex [41-46] Further, there are several lines of evidence that patients with $\mathrm{SCZ}$ had reduced $\mathrm{GABA}_{\mathrm{A}}$ receptormediated inhibition $[30,47,48]$ compared to healthy YNG group.

Again, as shown in Figure 2A, the amplitude of N45 component of spTMS was smaller in the OLD group and SCZ group (especially significantly lower in the OLD group) than in the YNG group, suggesting a relative decrease in $\mathrm{GABA}_{\mathrm{A}}$ receptor-mediated neurophysiological function. Thus, the decline in inhibitory neurophysiological function due to age-related changes as well as the pathophysiology of schizophrenia may affect the impairment of coordinated motor skills among motor control [49].

In addition, the multiple regression analysis demonstrated that P30 and N45 components are the significant factors to classify the groups. Interestingly, since P30 and N45 TEP components are supposed to be generated by $\mathrm{GABA}_{A}$ receptor-mediated function $[7,11,35,50]$, it is likely that $\mathrm{GABA}_{\mathrm{A}}$ ergic inhibition in the cortex is essential to maintain normal brain function.

Moreover, in terms of the response of TEP latencies after spTMS, since N45 and P60 are thought to be linked to $\mathrm{GABA}_{\mathrm{A}}$ and $\mathrm{GABA}_{\mathrm{B}}$ receptor-mediated functions as well as its excitatory function, respectively $[13,35]$, our finding of delayed response of P60 latency at the LC ROI by M1-spTMS

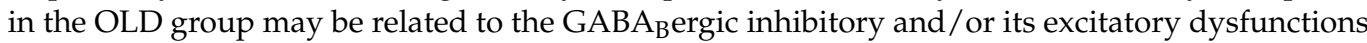
due to aging $[39,40]$, whereas the findings of delayed responses of N45 and P60 latencies at the midline central and right central ROIs after DLPFC-spTMS in the SCZ group may be associated with $\mathrm{GABA}_{\mathrm{A}}$ (i.e., N45) and $\mathrm{GABA}_{\mathrm{B}}$ (i.e., P60) receptor-mediated inhibitory and/or its excitatory (i.e., P60) dysfunctions [13,35]. Thus, these results might reflect conduction delays in both inhibitory and excitatory circuits, and that together these delayed responses of N45 and P60 might contribute to disrupted network connectivity in SCZ. Specifically, recent studies also suggest that a latency of P60 may be a correlate of neuronal excitability in M1 [13] and the DLPFC [51,52]. This reflects a larger distribution of cortical areas possibly due to more widespread neuropathological involvement related to the illness [43-46]. Indeed, we observed significantly delayed responses of N45-P60 latencies over the right central ROI even in the OLD group. However, the SCZ group demonstrated abnormal TEP latencies across short cortical distances compared to the OLD group (Figure 3), which may represent greater GABAergic inhibition deficits in the SCZ group.

Further, only the SCZ group showed a significantly delayed response of N45 latency over the right central ROI after DLPFC-spTMS. This finding may indicate more widespread GABA $\mathrm{A}_{\mathrm{A}}$ ergic inhibitory dysfunction possibly due to the functional disconnection between the left DLPFC and right central ROI in the SCZ [53]. Consistent with this contention, a previous functional magnetic resonance imaging study in SCZ demonstrated the functional hypoconnectivity in the somatomotor network [53], which may support our above finding of the delayed response of N45 in the SCZ group.

There are some limitations in the present study. First, the number of participants for each group was relatively small, although comparable to that of many previous TMS studies [7,35]. Thus, 
the findings warrant further research with larger sample sizes. Second, since we did not apply the auditory masking system during the experiments, the effect of auditory evoked potentials due to the conduction of the TMS click sounds on TEPs cannot be completely excluded [54]. Therefore, for future TMS-EEG studies, auditory white noise should be used during TMS stimulation as masking of the TMS click sound. Third, since patients with schizophrenia took medications when they participated in this study, there may be some medication effects on TEPs. Therefore, it would be necessary to more strictly control the influence of medications in the future study.

\section{Conclusions}

The present study demonstrated that TEP responses on amplitude and latency induced by spTMS have potential clinical application as a feasible neurophysiological probe to examine cortical functions in healthy people and patients with neuropsychiatric disorders such as SCZ and to assess the therapeutic effects of various interventions on TEP responses. Further, between the OLD and the SCZ groups, there were similarities in TEP responses in amplitude while there was a difference in TEP latency when looking at the propagation after the DLPFC-spTMS. To this end, our findings warrant further research in a larger sample size as well as in other neuropsychiatric disorders.

Supplementary Materials: The following are available online at https:/ / www.mdpi.com/2075-442 6/11/1/54/s1, Figure S1: Representative TEP waveforms at the left DLPFC obtained by spTMS for the YNG, OLD, and SCZ groups.

Author Contributions: Conceptualization, Y.N.; methodology, Y.N., R.Z., and P.L.; software, R.Z.; validation, Y.N., Z.J.D., and D.M.B.; formal analysis, Y.N. and R.Z.; investigation, Y.N., M.S.B., and R.F.H.C.; writing: original draft preparation, Y.N.; writing: review and editing, M.S.B., D.M.B., and P.L.; visualization, Y.N.; supervision, D.M.B., Z.J.D., and R.C.; project administration, Y.N., R.F.H.C., and M.S.B.; funding acquisition, D.M.B. All authors have read and agreed to the published version of the manuscript.

Funding: Temerty Centre for Therapeutic Brain Intervention and the Campbell Family Research Institute through the CAMH Foundation, and the Canada Foundation for Innovation.

Institutional Review Board Statement: The study was conducted according to the guidelines of the Declaration of Helsinki, and approved by the Institutional Review Board of the Centre for Addiction and Mental Health (protocol code: 003-2009; date of approval: 1 April 2014).

Informed Consent Statement: Informed consent was obtained from all subjects involved in the study.

Data Availability Statement: Data sharing not applicable. No new data were created or analyzed in this study. Data sharing is not applicable to this article.

Acknowledgments: This research was supported by the Temerty Centre for Therapeutic Brain Intervention, the Campbell Family Research Institute through the CAMH Foundation, and the Canada Foundation for Innovation.

Conflicts of Interest: None of the authors declare any conflicts of interest. Y.N. has received a Grantin-Aid for Young Scientists (KAKENHI) from the Japan Society for the Promotion of Science (JSPS), research grants from Japan Agency for Medical Research and Development (AMED), investigatorinitiated clinical study grants from TEIJIN PHARMA LIMITED and Inter Reha Co., Ltd. Y.N. also receives research grants from Japan Health Foundation, Meiji Yasuda Mental Health Foundation, Mitsui Life Social Welfare Foundation, Takeda Science Foundation, SENSHIN Medical Research Foundation, Health Science Center Foundation, Mochida Memorial Foundation for Medical and Pharmaceutical Research, Taiju Life Social Welfare Foundation, and Daiichi Sankyo Scholarship Donation Program. Y.N. has received speaker's honoraria from Dainippon Sumitomo Pharma, MOCHIDA PHARMACEUTICAL CO., Ltd., and Yoshitomiyakuhin Corporation within the past three years. Y.N. also receives equipment-in-kind support for an investigator-initiated study from Magventure Inc, Inter Reha Co., Ltd., Rogue Resolutions Ltd., and Miyuki Giken Co., Ltd. R.F.H.C. was supported by a Canadian Institutes of Health Research (CIHR)—Dystonia Medical Research Foundation Fellowship award. M.S.B. receives research support from the Brain and Behavior Research Foundation (Formerly NARSAD) Young Investigator Grant, Schizophrenia Junior Faculty Grant from the CAMH Foundation, and funding through the Excellence Fund, Department of Psychiatry, University of Toronto. R.C. received research support from the Canadian Institutes of Health Research (FDN 154292) and the National Institutes of Health (NS106822). Z.J.D. has received research support 
from the Ontario Mental Health (OMH) Foundation, the CIHR, the Brain and Behaviour Research Foundation (Formerly NARSAD), and the Temerty family and Grant family through the CAMH Foundation and the Campbell Institute. In the last 3 years, Z.J.D. has received research and equipment in-kind support for an investigator-initiated study through Brainsway Inc and Magventure Inc. His work was supported by the Ontario Mental Health Foundation (OMHF), the Canadian Institutes of Health Research (CIHR), the National Institutes of Mental Health and the Temerty Family and Grant Family and through the Centre for Addiction and Mental Health (CAMH) Foundation and the Campbell Institute. D.M.B. has received research support from the CIHR, NIH, Brain Canada and the Temerty Family through the CAMH Foundation and the Campbell Research Institute. He receives research support and in-kind equipment support for an investigator-initiated study from Brainsway Ltd. and he is the site principal investigator for three sponsor-initiated studies for Brainsway Ltd. He receives in-kind equipment support from Magventure for an investigator-initiated study. He receives medication supplies for an investigator-initiated trial from Indivior.

\author{
Abbreviations \\ SpTMS single-pulse transcranial magnetic stimulation \\ M1 primary motor cortex \\ DLPFC dorsolateral prefrontal cortex \\ TEP TMS-evoked potential
}

\title{
References
}

1. Ilmoniemi, R.J.; Virtanen, J.; Ruohonen, J.; Karhu, J.; Aronen, H.J.; Näätänen, R.; Katila, T. Neuronal responses to magnetic stimulation reveal cortical reactivity and connectivity. Neuroreport 1997, 8, 3537-3540. [CrossRef] [PubMed]

2. Daskalakis, Z.J.; Farzan, F.; Barr, M.S.; Maller, J.J.; Chen, R.; Fitzgerald, P.B. Long-interval cortical inhibition from the dorsolateral prefrontal cortex: A TMS-EEG study. Neuropsychopharmacology 2008, 33, 2860-2869. [CrossRef] [PubMed]

3. Farzan, F.; Barr, M.S.; Wong, W.; Chen, R.; Fitzgerald, P.B.; Daskalakis, Z.J. Suppression of gamma-oscillations in the dorsolateral prefrontal cortex following long interval cortical inhibition: A TMS-EEG study. Neuropsychopharmacology 2009, 34, $1543-1551$. [CrossRef] [PubMed]

4. Fitzgerald, P.B.; Maller, J.J.; Hoy, K.; Farzan, F.; Daskalakis, Z.J. GABA and cortical inhibition in motor and non-motor regions using combined TMS-EEG: A time analysis. Clin. Neurophysiol. 2009, 120, 1706-1710. [CrossRef]

5. Fuggetta, G.; Fiaschi, A.; Manganotti, P. Modulation of cortical oscillatory activities induced by varying single-pulse transcranial magnetic stimulation intensity over the left primary motor area: A combined EEG and TMS study. Neuroimage 2005, 27, 896-908. [CrossRef]

6. Paus, T.; Sipila, P.K.; Strafella, A.P. Synchronization of neuronal activity in the human primary motor cortex by transcranial magnetic stimulation: An EEG study. J. Neurophysiol. 2001, 86, 1983-1990. [CrossRef]

7. Premoli, I.; Castellanos, N.; Rivolta, D.; Belardinelli, P.; Bajo, R.; Zipser, C.; Espenhahn, S.; Heidegger, T.; Müller-Dahlhaus, F.; Ziemann, U. TMS-EEG signatures of GABAergic neurotransmission in the human cortex. J. Neurosci. 2014, 34, 5603-5612. [CrossRef]

8. Rogasch, N.C.; Daskalakis, Y.J.; Fitzgerald, P.B. Cortical inhibition of distinct mechanisms in the dorsolateral prefrontal cortex is related to working memory performance: A TMS-EEG study. Cortex 2015, 64, 68-77. [CrossRef]

9. Bonato, C.; Miniussi, C.; Rossini, P.M. Transcranial magnetic stimulation and cortical evoked potentials: A TMS/EEG coregistration study. Clin. Neurophysiol. 2006, 117, 1699-1707. [CrossRef]

10. Lioumis, P.; Kičić, D.; Savolainen, P.; Mäkelä, J.P.; Kähkönen, S. Reproducibility of TMS-evoked EEG responses. Hum. Brain Mapp. 2009, 30, 1387-1396. [CrossRef]

11. Ferreri, F.; Rossini, P.M. TMS and TMS-EEG techniques in the study of the excitability, connectivity, and plasticity of the human motor cortex. Rev. Neurosci. 2013, 24, 431-442. [CrossRef] [PubMed]

12. Komssi, S.; Kahkonen, S. The novelty value of the combined use of electroencephalography and transcranial magnetic stimulation for neuroscience research. Brain. Res. Rev. 2006, 52, 183-192. [CrossRef] [PubMed]

13. Cash, R.F.; Noda, Y.; Zomorrodi, R.; Radhu, N.; Farzan, F.; Rajji, T.K.; Fitzgerald, P.B.; Chen, R.; Daskalakis, Z.J.; Blumberger, D.M. Characterization of glutamatergic and GABAA-Mediated neurotransmission in motor and dorsolateral prefrontal cortex using paired-pulse TMS-EEG. Neuropsychopharmacology 2017, 42, 502-511. [CrossRef] [PubMed]

14. Komssi, S.; Kahkonen, S.; Ilmoniemi, R.J. The effect of stimulus intensity on brain responses evoked by transcranial magnetic stimulation. Hum. Brain Mapp. 2004, 21, 154-164. [CrossRef]

15. Kahkonen, S.; Komssi, S.; Wilenius, J.; Ilmoniemi, R.J. Prefrontal transcranial magnetic stimulation produces intensity-dependent EEG responses in humans. Neuroimage 2005, 24, 955-960. [CrossRef]

16. Rosanova, M.; Casali, A.; Bellina, V.; Resta, F.; Mariotti, M.; Massimini, M. Natural frequencies of human corticothalamic circuits. J. Neurosci. 2009, 29, 7679-7685. [CrossRef]

17. Kahkonen, S.; Wilenius, J.; Komssi, S.; Ilmoniemi, R.J. Distinct differences in cortical reactivity of motor and prefrontal cortices to magnetic stimulation. Clin. Neurophysiol. 2004, 115, 583-588. [CrossRef] 
18. Kahkonen, S.; Komssi, S.; Wilenius, J.; Ilmoniemi, R.J. Prefrontal TMS produces smaller EEG responses than motor-cortex TMS: Implications for rTMS treatment in depression. Psychopharmacology 2005, 181, 16-20. [CrossRef]

19. Casarotto, S.; Herukka, S.; Pigorini, A.; Napolitani, M.; Gosseries, O.; Niskanen, E.; Könönen, M.; Mervaala, E.; Rosanova, M.; Soininen, H.; et al. Transcranial magnetic stimulation-evoked EEG/cortical potentials in physiological and pathological aging. Neuroreport 2011, 22, 592-597. [CrossRef]

20. Levit-Binnun, N.; Litvak, V.; Pratt, H.; Moses, E.; Zaroor, M.; Peled, A. Differences in TMS-evoked responses between schizophrenia patients and healthy controls can be observed without a dedicated EEG system. Clin. Neurophysiol. 2010, 121, 332-339. [CrossRef]

21. Ferrarelli, F.; Massimini, M.; Peterson, M.J.; Riedner, B.A.; Lazar, M.; Murphy, M.J.; Huber, R.; Rosanova, M.; Alexander, A.L.; Kalin, N.; et al. Reduced evoked gamma oscillations in the frontal cortex in schizophrenia patients: A TMS/EEG study. Am. J. Psychiatry 2008, 165, 996-1005. [CrossRef] [PubMed]

22. Bagarinao, E.; Watanabe, H.; Maesawa, S.; Mori, D.; Hara, K.; Kawabata, K.; Yoneyama, N.; Ohdake, R.; Imai, K.; Masuda, M.; et al. An unbiased data-driven age-related structural brain parcellation for the identification of intrinsic brain volume changes over the adult lifespan. Neuroimage 2018, 169, 134-144. [CrossRef] [PubMed]

23. Konopaske, G.T.; Lange, N.; Coyle, J.T.; Benes, F.M. Prefrontal cortical dendritic spine pathology in schizophrenia and bipolar disorder. JAMA Psychiatry 2014, 71, 1323-1331. [CrossRef] [PubMed]

24. Wang, S.; Young, K.M. White matter plasticity in adulthood. Neuroscience 2014, 276, 148-160. [CrossRef] [PubMed]

25. Peters, A. The effects of normal aging on myelin and nerve fibers: A review. J. Neurocytol. 2002, 31, 581-593. [CrossRef]

26. Pajevic, S.; Basser, P.J.; Fields, R.D. Role of myelin plasticity in oscillations and synchrony of neuronal activity. Neuroscience 2014, 276, 135-147. [CrossRef]

27. Noda, Y. Toward the establishment of neurophysiological indicators for neuropsychiatric disorders using transcranial magnetic stimulation-evoked potentials: A systematic review. Psychiatry Clin. Neurosci. 2020, 74, 12-34. [CrossRef]

28. Maatta, S.; Könönen, M.; Kallioniemi, E.; Lakka, T.; Lintu, N.; Lindi, V.; Ferreri, F.; Ponzo, D.; Säisänen, L. Development of cortical motor circuits between childhood and adulthood: A navigated TMS-HdEEG study. Hum. Brain Mapp. 2017, 38, 2599-2615. [CrossRef]

29. Noda, Y.; Zomorrodi, R.; Cash, R.F.H.; Barr, M.S.; Farzan, F.; Rajji, T.K.; Chen, R.; Daskalakis, Z.J.; Blumberger, D.M. Characterization of the influence of age on GABAA and glutamatergic mediated functions in the dorsolateral prefrontal cortex using paired-pulse TMS-EEG. Aging 2017, 9, 556-572. [CrossRef]

30. Noda, Y.; Barr, M.S.; Zomorrodi, R.; Cash, R.F.H.; Farzan, F.; Rajji, T.K.; Chen, R.; Daskalakis, Z.J.; Blumberger, D.M. Evaluation of short interval cortical inhibition and intracortical facilitation from the dorsolateral prefrontal cortex in patients with schizophrenia. Sci. Rep. 2017, 7, 17106. [CrossRef]

31. Chung, S.W.; Rogasch, N.C.; Hoy, K.E.; Fitzgerald, P.B. Measuring brain stimulation induced changes in cortical properties using TMS-EEG. Brain Stimul. 2015, 8, 1010-1020. [CrossRef] [PubMed]

32. McClintock, S.M.; Freitas, C.; Oberman, L.; Lisanby, S.H.; Pascual-Leone, A. Transcranial magnetic stimulation: A neuroscientific probe of cortical function in schizophrenia. Biol. Psychiatry 2011, 70, 19-27. [CrossRef] [PubMed]

33. Davies, C.H.; Davies, S.N.; Collingridge, G.L. Paired-pulse depression of monosynaptic GABA-mediated inhibitory postsynaptic responses in rat hippocampus. J. Physiol. 1990, 424, 513-531. [CrossRef] [PubMed]

34. Deisz, R.A. GABA(B) receptor-mediated effects in human and rat neocortical neurones In Vitro. Neuropharmacology 1999, 38, 1755-1766. [CrossRef]

35. Ferreri, F.; Pasqualetti, P.; Määttä, S.; Ponzo, D.; Ferrarelli, F.; Tononi, G.; Mervaala, E.; Miniussi, C.; Rossini, P.M. Human brain connectivity during single and paired pulse transcranial magnetic stimulation. Neuroimage 2011, 54, 90-102. [CrossRef]

36. Peinemann, A.; Lehner, C.; Conrad, B.; Siebner, H.R. Age-related decrease in paired-pulse intracortical inhibition in the human primary motor cortex. Neurosci. Lett. 2001,313, 33-36. [CrossRef]

37. Marneweck, M.; Loftus, A.; Hammond, G. Short-interval intracortical inhibition and manual dexterity in healthy aging. Neurosci. Res. 2011, 70, 408-414. [CrossRef]

38. Heise, K.F.; Zimerman, M.; Hoppe, J.; Gerloff, C.; Wegscheider, K.; Hummel, F.C. The aging motor system as a model for plastic changes of GABA-mediated intracortical inhibition and their behavioral relevance. J. Neurosci. 2013, 33, 9039-9049. [CrossRef]

39. Grachev, I.D.; Apkarian, A.V. Aging alters regional multichemical profile of the human brain: An. in vivo 1H-MRS study of young versus middle-aged subjects. J. Neurochem. 2001, 76, 582-593. [CrossRef]

40. Grachev, I.D.; Swarnkar, A.; Szeverenyi, N.M.; Ramachandran, T.S.; Apkarian, A.V. Aging alters the multichemical networking profile of the human brain: An. In Vivo (1)H-MRS study of young versus middle-aged subjects. J. Neurochem. 2001, 77, 292-303. [CrossRef]

41. Benes, F.M.; McSparren, J.; Bird, E.D.; SanGiovanni, J.P.; Vincent, S.L.B. Deficits in small interneurons in prefrontal and cingulate cortices of schizophrenic and schizoaffective patients. Arch. Gen. Psychiatry 1991, 48, 996-1001. [CrossRef] [PubMed]

42. Benes, F.M.; Kwok, E.W.; Vincent, S.L.; Todtenkopf, M.S. A reduction of nonpyramidal cells in sector CA $\mathrm{C}_{2}$ of schizophrenics and manic depressives. Biol. Psychiatry 1998, 44, 88-97. [CrossRef]

43. Woo, T.U.; Walsh, J.P.; Benes, F.M. Density of glutamic acid decarboxylase 67 messenger RNA-containing neurons that express the N-methyl-D-aspartate receptor subunit NR2A in the anterior cingulate cortex in schizophrenia and bipolar disorder. Arch. Gen. Psychiatry 2004, 61, 649-657. [CrossRef] [PubMed] 
44. Lewis, D.A.; Hashimoto, T.; Volk, D.W. Cortical inhibitory neurons and schizophrenia. Nat. Rev. Neurosci. 2005, 6, 312-324. [CrossRef] [PubMed]

45. Akbarian, S.; Huang, H.S. Molecular and cellular mechanisms of altered GAD1/GAD67 expression in schizophrenia and related disorders. Brain Res. Rev. 2006, 52, 293-304. [CrossRef]

46. Benes, F.M.; Lim, B.; Matzilevich, D.; Walsh, J.P.; Subburaju, S.; Minns, M. Regulation of the GABA cell phenotype in hippocampus of schizophrenics and bipolars. Proc. Natl. Acad. Sci. USA 2007, 104, 10164-10169. [CrossRef] [PubMed]

47. Takahashi, S.; Ukai, S.; Kose, A.; Hashimoto, T.; Iwatani, J.; Okumura, M.; Tsuji, T.; Shinosaki, K. Reduction of cortical GABAergic inhibition correlates with working memory impairment in recent onset schizophrenia. Schizophr. Res. 2013, 146, 238-243. [CrossRef]

48. Bridgman, A.C.; Barr, M.S.; Goodman, M.S.; Chen, R.; Rajji, T.K.; Daskalakis, Z.J.; George, T.P. Deficits in GABAA receptor function and working memory in non-smokers with schizophrenia. Schizophr. Res. 2016, 171, 125-130. [CrossRef]

49. Umesawa, Y.; Matsushima, K.; Atsumi, T.; Kato, T.; Fukatsu, R.; Wada, M.; Ide, M. Altered GABA concentration in brain motor area is associated with the severity of motor disabilities in individuals with autism spectrum disorder. J. Autism Dev. Disord. 2020, 50, 2710-2722. [CrossRef]

50. Gray, D.T.; Engle, J.R.; Rudolph, M.L.; Recanzone, G.H. Regional and age-related differences in GAD67 expression of parvalbuminand calbindin-expressing neurons in the rhesus macaque auditory midbrain and brainstem. J. Comp. Neurol. 2014, 522, 4074-4084. [CrossRef]

51. Hill, A.T.; Rogasch, N.C.; Fitzgerald, P.B.; Hoy, K.E. TMS-EEG: TMS-EEG: A window into the neurophysiological effects of transcranial electrical stimulation in non-motor brain regions. Neurosci. Biobehav. Rev. 2016, 64, 175-184. [CrossRef] [PubMed]

52. Chung, S.W.; Sullivan, C.M.; Rogasch, N.C.; Hoy, K.E.; Bailey, N.W.; Cash, R.F.H.; Fitzgerald, P.B. The effects of individualised intermittent theta burst stimulation in the prefrontal cortex: A TMS-EEG study. Hum. Brain Mapp. 2019, 40, 608-627. [CrossRef] [PubMed]

53. Li, M.; Deng, W.; He, Z.; Wang, Q.; Huang, C.; Jiang, L.; Gong, O.; Ziedonis, D.M.; King, J.A.; Ma, X.; et al. A splitting brain: Imbalanced neural networks in schizophrenia. Psychiatry Res. 2015, 232, 145-153. [CrossRef] [PubMed]

54. Rocchi, L.; Di Santo, A.; Brown, K.; Ibáñez, J.; Casula, E.; Rawji, V.; Di Lazzaro, V.; Koch, G.; Rothwell, J. Disentangling EEG responses to TMS due to cortical and peripheral activations. Brain Stimul. 2020, 14, 4-18. [CrossRef] 